\title{
Estado Nutricional em Idosos com Doenças Crônicas não Transmissíveis
}

\author{
Nutritional State in Seniors With Chronic non Communicable Diseases
}

Estado Nutricional en Ancianos con Enfermedades Crónicas no Transmisibles

\author{
Wellington Danilo Soares ${ }^{1}$ \\ Priscila Silva Rocha ${ }^{2}$ \\ Jéssica Pereira Barbosa ${ }^{3}$ \\ Priscilla Kálisy Duarte Soares ${ }^{4}$ \\ Daniel Antunes Freitas ${ }^{5}$
}

\section{Resumo}

Objetivo: O objetivo deste estudo foi analisar o estado nutricional de idosos portadores de Doenças Crônicas Não Transmissíveis (DCNT) de um centro de referência no tratamento de idosos. Método: Trata-se de uma pesquisa descritiva, com abordagem quantitativa e transversal. Compôs a amostra 50 indivíduos, de ambos os sexos, faixa etária acima de 60 anos, selecionados de forma aleatória todos idosos atendidos em um centro de referência em idosos na cidade de Montes Claros - MG. Para a coleta de dados das Doenças Crônicas Não Transmissíveis foi utilizado um questionário baseado no Standard Health Questionare (SHQ) e adaptado, já para avaliação do estado nutricional foi utilizado o protocolo de Índice de Massa Corporal (IMC) e Circunferência da Cintura (CC). Resultados: Os resultados demonstraram que a maioria dos avaliados (82\%) apresentaram hipertensão arterial, associada a outra doença ou não, $70 \%$ eram do sexo feminino, sendo que grande parte dos participantes apresentaram estados nutricionais inadequados

\footnotetext{
${ }^{1}$ Doutorado no Programa de Pós-graduação em Ciências da Saúde. Universidade Estadual de Montes Claros, Unimontes. Montes Claros, MG, Brasil. Autor correspondente: Avenida Ruy Braga, s/n - Vila Mauriceia Montes Claros - MG.E-mail: wdansoa@yahoo.com.br ${ }^{2}$ Graduanda do Curso de Nutrição. Faculdade de Saúde Ibituruna, FASI/Soebras. Montes Claros, MG, Brasil. ${ }^{3}$ Graduanda do Curso de Nutrição. Faculdade de Saúde Ibituruna, FASI/Soebras. Montes Claros, MG, Brasil. ${ }^{4}$ Programa de Pós-graduação em Enfermagem e Neonatal. Instituto Fernandes Figueira, Rio de Janeiro,Brasil. ${ }^{5}$ Doutorado no Programa de Pós-graduação em Ciências da Saúde. Universidade Estadual de Montes Claros, Unimontes. Montes Claros, MG, Brasil.
} 
sobrepeso e (28\%) baixo peso. Em relação a CC, $68 \%$ apresentaram valores acima do recomendado, indicando maior risco de doenças cardiovasculares. Conclusão: Conclui-se, portanto que os idosos com estados nutricionais inadequados são mais propícios a desenvolver doenças crônicas.

\section{Palavras-chaves: Idosos; Doenças Crônicas; Estado Nutricional.}

\section{Abstract}

Objective: The objective of this study was to analyze the nutritional state of senior bearers of DCNT of a reference center in the treatment of senior. Method: It is a descriptive research, with quantitative and traverse approach. He/she composed the sample 50 individuals, both sexes, age group above 60 years, selected in a random way all senior ones assisted in a reference center in seniors in the city of Montes Claros-MG. For the collection of data of DCNT a questionnaire was used based on the Standard Health Questionare (SHQ) and adapted, already for evaluation of the nutritional state it was used the protocol of Index of Corporal (IMC) Mass and Circumference of the Waist (CC). Results: The results demonstrated that most of the appraised (82\%) ones presented hypertension arterial, associated the other disease or no, (34\%) nutritional overweight and (28\%) low weight. In relation to $C C, 68 \%$ presented values above recommended him/it, indicating larger risk of cardiovascular diseases. Conclusion: It is ended, therefore that the seniors with inadequate nutritional states are more favorable to develop chronic diseases.

Keywords: Senior; Chronic Diseases; Nutritional State.

\section{Resumen}

Objetivo: El objetivo de este estudio fue analizar el estado nutricional de ancianos portadores de Enfermedades Crónicas No Transmisibles (ECNT) de un centro de referencia en el tratamiento de ancianos. Método: se trata de una investigación descriptiva, con un abordaje cuantitativo y transversal. La muestra fue compuesta por 50 individuos, ambos sexos, grupo de edad mayor de 60 años, seleccionados aleatoriamente, todos atendidos en un centro de referencia en ancianos en la ciudad de Montes Claros-MG. Para la recolecta de datos de las Enfermedades Crónicas No Transmisibles fue utilizado 
un cuestionario basado en el Standard Health Questionare (SHQ) y adaptado, para la evaluación del estado nutricional fue utilizado el protocolo de Índice de Masa Corporal (IMC) y Circunferencia de la Cintura (CC). Resultados: los resultados mostraron que la mayoría de los evaluados (82\%) presentaron hipertensión arterial asociada a otra enfermedad o no, $70 \%$ eran del sexo femenino, siendo que gran parte de los participantes presentaron estado nutricional no adecuado (34\%) sobrepeso y (28\%) peso bajo. Con relación a la CC, $68 \%$ presentaron valores más de lo recomendable, indicando risco más grande de enfermedades cardiovasculares. Conclusión: se puede concluir, por lo tanto, que los ancianos en estados nutricionales no adecuados son más propicios a desarrollar enfermedades crónicas.

Palabras clave: Ancianos; Enfermedades Crónicas; Estado Nutricional.

\section{Introdução}

Envelhecer faz parte do ciclo natural da vida, e atualmente é possível envelhecer de forma saudável, com aptidões físicas e funcionais, no entanto também é possível que este grupo etário se torne enfermo devido aos processos fisiológicos naturais do corpo, acarretando uma dependência medicamentosa e física ${ }^{(1)}$.

Os indivíduos com faixa etária igual ou superior a 60 anos são idosos ${ }^{(2)}$. $\mathrm{O}$ aumento no número de idosos exige mais pesquisas para averiguar a prevalência de desordens nutricionais, favorecendo cuidado e melhor atenção à velhice ${ }^{(3)}$.

No Brasil, as Doenças Crônicas Não Transmissíveis (DCNT) são as principais causas de mortalidade tanto na população adulta como na idosa, destacando-se as doenças cardiovasculares e as neoplasias $^{(4)}$.

As DCNT e suas predisposições comprometem independentemente da situação socioeconômica, aqueles mais susceptíveis, como os de idade avançada, com rendas inferiores e com grau de educação menor ${ }^{(5)}$. Seu aparecimento está vinculado ao envelhecimento, estilo de vida, tabagismo, hábitos alimentares inadequados, consumo de álcool, inatividade física, comportamento sexual de risco e predisposição genética $^{(6)}$.

A população brasileira enfrenta mudança nutricional desfavorável, visto que os alimentos naturais vêm sendo 
substituídos pelos alimentos industrializados, ricos em sódio, gorduras e substâncias químicas, compondo assim, uma alimentação inadequada e pobre em nutrientes ${ }^{(7)}$. Para que não ocorram desordens funcionais e por consequência $\mathrm{o}$ surgimento das patologias práticas alimentares saudáveis são fundamentais $^{(8)}$. Há muitos idosos que não seguem nenhuma dieta, ingerem demasiadamente gorduras e carboidratos aliado a inatividade física ${ }^{(9)}$, deve-se ter também preocupação com o baixo peso.

Com o crescimento da expectativa de vida, é de grande valor o desenvolvimento de qualidade de vida onde a reeducação alimentar, prevenção e manutenção visam à promoção de um melhor estado de saúde ${ }^{(10,11)}$. Manter um estado nutricional adequado na terceira idade é difícil. Neste contexto objetivou-se analisar o estado nutricional de idosos portadores de DCNT de um centro de referência no tratamento de idosos na cidade de Montes Claros - MG.

\section{Método}

A pesquisa é de caráter descritivo com abordagem quantitativa, transversal (12). A amostra foi de 50 idosos acima de 60 anos, de ambos os sexos, todos pacientes regulares de um hospital de referência para idosos da cidade de Montes Claros. Foram incluídos todos idosos presentes no dia da coleta de dados e que aceitaram participar da pesquisa, excluídos aqueles que não eram portadores de DCNT.

$\mathrm{Na}$ avaliação do estado nutricional foi utilizado o protocolo de Índice de Massa Corporal (IMC), que consiste na razão entre peso corporal $(\mathrm{kg})$ e estatura (m) ao quadrado, utilizando-se os pontos de cortes preconizados (13). Para a mensuração do peso corporal foi utilizado uma balança digital da marca OMRON com plataforma de vidro e capacidade de $150 \mathrm{~kg}$, precisão de 0,10 gramas. $\mathrm{Na}$ aferição da estatura um estadiômetro da marca SECA com capacidade de 2,2 metros e precisão de 0,1 centímetros.

As pesquisadoras aplicaram os questionários e coletaram os dados dos participantes em uma sala reservada com intuito de preservar o sigilo dos dados e privacidade dos envolvidos, todas as avaliações foram realizadas no mês de março de 2016.

Após a coleta de dados, foram feitas análises descritivas com valores de frequência, porcentagem, média e desvio padrão. Todo o procedimento estatístico foi realizado através do 
Statistical Package for the Social Sciences (SPSS) versão 22.0 para Windows.

$\mathrm{O}$ estudo foi submetido e aprovado pelo Comitê de Ética em Pesquisa (CEP) da Associação Educativa do Brasil - SOEBRAS, sob o parecer $\mathrm{n}^{\circ} 1.419 .130$.

\section{Resultados}

O presente estudo avaliou 50 idosos, sendo $70 \%$ do sexo feminino e $30 \%$ do sexo masculino, com idade média de 73 anos. Com relação à classificação do risco de comorbidades, as medidas de circunferência da cintura (CC) apresentaram 58\% das idosas com valores elevados ( $\geq 80 \mathrm{~cm})$ e os idosos apenas $10 \%(\geq 94 \mathrm{~cm})$. Enquanto que $32 \%$ apresentaram valores adequados de CC. Das mulheres com $\mathrm{CC} \geq 80 \mathrm{~cm}$, $37 \%$ delas estavam com sobrepeso. Quanto aos homens com CC $\geq 94 \mathrm{~cm}$, $27 \%$ sobrepeso.

Tabela 1 - Apresenta a caracterização do grupo amostral $(\mathbf{n}=50)$

\begin{tabular}{cccc}
\hline VARIÁVEL & MINIMO & MÁXIMO & MÉDIA E DP \\
\hline Idade & 61 & 95 & $73,5 \pm 7,8$ \\
IMC & 13,7 & 36,8 & $24,7 \pm 5,0$ \\
CC & 67 & 127 & $92,3 \pm 13,3$ \\
\hline
\end{tabular}

Os resultados da aferição da $\mathrm{CC}$ evidenciam que entre os estados nutricionais dos idosos avaliados, $68 \%$ apresentaram valores de CC acima do recomendado, o que aumenta o risco de doenças cardiovasculares dentre outras possíveis enfermidades futuras ${ }^{(14)}$. Na classificação do estado nutricional através do IMC, 38\% dos idosos apresentaram eutrofia, $34 \%$ foram classificados com sobrepeso e $28 \%$ com baixo peso.

Tabela 2 - Demonstra os resultados referentes à classificação nutricional dos avaliados com valores de frequência e porcentagem.

\begin{tabular}{ccc}
\hline VARIÁVEL & FREQUÊNCIA & PORCENTAGEM (\%) \\
\hline Baixo peso & 14 & 28 \\
Eutrofia & 19 & 38 \\
Sobrepeso & 17 & 34 \\
\hline
\end{tabular}


a presença de patologias ${ }^{(15)}$. Entre os dados obtidos na pesquisa, as patologias mais identificadas foram a hipertensão arterial (82\%), diabetes (24\%) e osteoporose (42\%). Entretanto alguns destes indivíduos avaliados apresentaram mais de uma patologia, representando o percentual de $58 \%$ da população de estudo. Dos idosos que foram identificados com mais de uma patologia $44,8 \%$ possuíam sobrepesos, $41,4 \%$ baixo peso e $13,8 \%$ eutrofia.

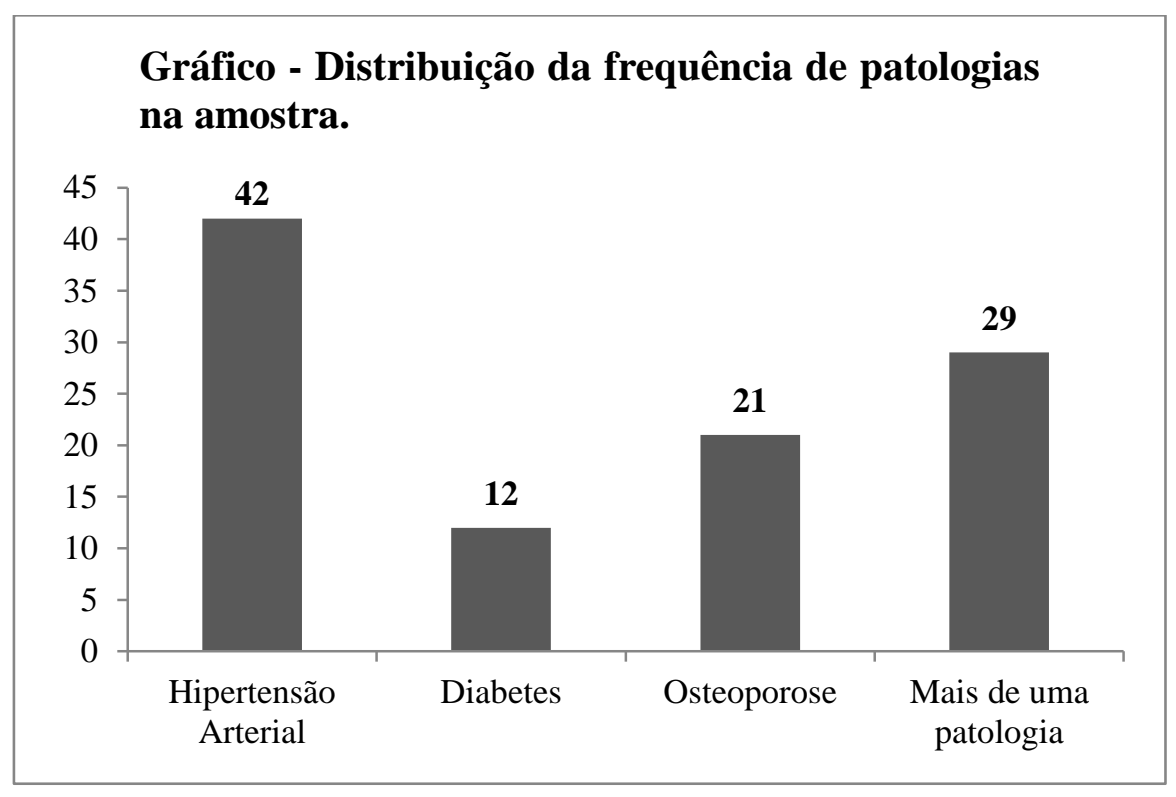

Associando as doenças com os estados nutricionais dos mesmos é possível perceber que aqueles que apresentam um estado nutricional inadequado estão mais propícios ao surgimento das doenças. Daqueles com hipertensão arterial, $41,5 \%$ eram classificados com sobrepeso e 19,5\% com baixo peso. Já aqueles com diabetes, $58,3 \%$ estavam com sobrepeso e $25 \%$ com baixo peso.

\section{Discussão}

O perfil nutricional da população analisada foi caracterizado pela incidência tanto de eutrofia (38\%) como sobrepeso (34\%). É extremamente importante a detecção dos indivíduos em risco nutricional, pois deste modo se torna possível realizar intervenção nutricional primária ${ }^{(16)}$.

A maior parte dos indivíduos eram do sexo feminino representando o percentual de $70 \%$, o que se subentende que as mulheres se preocupam mais com a saúde do que os homens. A Circunferência da Cintura (CC) apresentou níveis elevados em ambos os 
sexos, sendo detectada pelo acúmulo de gordura corporal, o mesmo acontece em um estudo de Covatti et $a .^{(17)}$ em que dos 284 pacientes avaliados $56 \%$ apresentaram $\mathrm{CC}$ elevada e consequentemente maior risco de doenças cardiovasculares.

Em relação as patologias, houve a prevalência de hipertensão arterial (82\%), diabetes mellitus (24\%) e osteoporose (42\%). Dos 82\% avaliados que apresentaram hipertensão arterial, $41,5 \%$ eram classificados como sobrepeso. Deste modo é possível dizer que o sobrepeso é um fator de risco para o aparecimento de Doenças Crônicas Não Transmissíveis, entre elas a hipertensão arterial e o diabetes mellitus ${ }^{(18)}$.

A Hipertensão Arterial Sistêmica (HAS) é uma doença comumente identificada entre os idosos, possui evidências de que alterações no processo de senescência fazem com que os indivíduos sejam mais propensos ao aparecimento da HAS, sendo, portanto, a principal patologia crônica nesta faixa etária além de ser fator de risco às doenças cardiovasculares ${ }^{(19)}$. Um estudo realizado em Natal - RN, estimou-se que do total da amostra (52 idosos) $55,8 \%(n=29)$ apresentaram hipertensão $\operatorname{arterial}^{(20)}$.
O diabetes é uma patologia séria, que causa restrições ao portador, merece cuidado e atenção especial e pode ser considerado um problema de saúde pública. É relevante o incentivo à promoção de saúde e os estilos de vida saudáveis, uma vez que estas resultam positivamente na qualidade de vida destes idosos diabéticos ${ }^{(21)}$. Em estudo em Roca Sales - RS com amostra de 112 indivíduos com idade acima de 60 anos, $23,2 \%(n=26)$ foram classificados com diabetes mellitus ${ }^{(22)}$.

A osteoporose é uma doença osteomuscular que acomete principalmente os idosos, é marcada pela perda gradativa de massa óssea, fazendo com que o osso fique mais frágil e susceptível a fraturas. Devido a essa fragilidade os indivíduos necessitam de prevenções clínicas e nutricionais a fim de evitar e/ou reduzir os fatores de risco ligados ao surgimento desta patologia ${ }^{(23)}$. Estudo realizado por Sardinha et $a l^{(24)}$ em um grupo amostral de 308 idosos, identificou-se $26,3 \%$ também com osteoporose.

Os estilos que são adotados no decorrer da vida refletem-se na senescência e as rotinas saudáveis têm a capacidade de transformar a velhice em uma fase agradável ${ }^{(25)}$. Porém vale ressaltar que todos os indivíduos, e não 
somente os idosos, necessitam de um acompanhamento primordial de saúde, para uma significativa saúde em sua terceira idade.

\section{Conclusão}

Com base neste estudo, a presente pesquisa permite-se concluir que níveis elevados do estado nutricional dos idosos entre as medidas de IMC e CC, constituindo para fatores de risco de desenvolvimento de Doenças Crônicas Não Transmissíveis, sendo que as mais diagnosticadas foram hipertensão arterial, diabetes e osteoporose. Diante da exposta pesquisa as mulheres foram mais abrangentes a essa avaliação, por preocuparem-se mais com a saúde, processo de envelhecimento e modificações fisiológicas do organismo. Portanto, é imprescindível estudos que visam avaliar o estado nutricional do grupo de idosos, com propósito de proporcionar variações positivas, com intervenções apropriadas, nos aspectos dietéticos e nutritivos, a fim de propiciar melhoria da qualidade de vida.

\section{Referências}

1. Floriano LA, Azevedo RCS, Reiners, AAO, Sudré MRS. Cuidado realizado pelo cuidador familiar ao idoso dependente, em domicílio, no contexto da estratégia de saúde da família. Texto Contexto Enferm. 2012; 21(3):543-8.

2. Ministério da Saúde. Portaria $\mathrm{n}^{\circ}$ 10.741, de 01 de outubro de 2003. Dispõe sobre o Estado do Idoso e dá outras providências. Disponível em: http://bvsms.saude.gov.br/bvs/publicaco es/estatuto_idoso_3edicao.pdf. Acesso em: 5 Setembro 2015.

3. Lehn F, Coelho HDS, Garcia MT, Scabar LF. Estado nutricional de idosos em uma instituição de longa permanência. J. Health Sci Inst. 2012;30(1):53-8.

4. Paulo TRS, Gomes IC, Santos VR, Destro DG, Cristofaro DGD, Castellano $\mathrm{SM}$, et al. Atividade física e estado nutricional: fator de proteção para Doenças Crônicas Não Transmissíveis (DCNT) em idosas? Rev Bras Promoç Saúde. 2014; 27(4):527-32.

5. Francisco PMSB, Segri NJ, Barros MBA, Malta DC. Desigualdades sociodemográficas nos fatores de risco e proteção para doenças crônicas não transmissíveis: inquérito telefônico em Campinas, São Paulo. Epidemiol. e Serv. Saúde. 2015; 24(1):7-8.

6. Veras RP. Um modelo em que todos ganham: mudar e inovar, desafios para o enfrentamento das doenças crônicas entre os idosos. Acta Scientiarum. 2012;34(1):3-8.

7. Brauner MCC, Furlan KM. O risco alimentar na contemporaneidade: Uma análise do quadro epidêmico da diabetes à luz dos princípios constitucionais para promoção da proteção ambiental em prol da saúde pública. Revista da AJURIS. 2014;41(135):289-15.

8. Monteiro MAM. Percepção sensorial dos alimentos em idosos. Revista Espaço para a Saúde. 2009; 10(2):34-2. 
9. Medeiros P, Lima RA, Sardinha AHL, Diniz DC, Aragão MAM. Aspectos nutricionais de idosos atendidos em um centro de saúde. Rev Pesq Saúde. 2014; 15 (3):351-5.

10. Dórea GS, Pina MGM, Santos D. Aspectos nutricionais de idosos praticantes de atividade física. Revista Demetra. 2015; 10(2):347-60.

11. Nascimento CM, Ribeiro AQ, San'Ana LFR, Oliveira RMS, Franceschini SCC, Priore SE. Estado nutricional e condições de saúde da população idosa brasileira: revisão de literatura. Revista Med Minas. 2011; 21(2):174-80.

12. Triviños A. Introdução à pesquisa em ciências sociais: a pesquisa qualitativa em educação. São Paulo: Atlas; 2009.

13. ADA. The Nutriton Screanning Initiative. Incorporating Nutrition Screening and Interventions into Medical Practice. A Monograph for Physicians. Washington D.C. US: American Academy of Family Physicians. The American Dietetic Association. National Council on Aging Inc; 1994. Disponível em http://www.jblearning.com/samples/076 3730 629/Frank_Appendix10D.pdf. Acesso em: 6 de Setembro 2015.

14. Previato HDRA, Dias APV, Nemer ASA, Nimer M. Associação entre o índice de massa corporal $\mathrm{e}$ circunferência da cintura em idosas, Ouro Preto, Minas Gerais, Brasil. Revista Nutr. Clí. Diet. Hosp. 2014; 34(1):25-0.

15. Cintra RMG, Oliveira D, Silva LMG. Estado nutricional e ocorrência de hipertensão arterial e de diabetes em idosos residentes em instituições geriátricas. Revista Alimentos e Nutrição Araraquara. 2012; 23(4):56775 .

16. Souza R, Fraga JS, Gottschall CBA, Busnello FM, Rabito EI. Avaliação antropométrica em idosos: estimativas de peso e altura e concordância entre classificações de IMC. Rev. Bras. Geriatr. e Gerontol. 2013; 16(1):81-0.

17. Covatti CF, Santos JM, Vicente AAS, Greff NT, Vicentini AP. Fatores de risco para doenças cardiovasculares em adultos e idosos de um hospital universitário. Revista Nutr. Clí. Diet. Hosp. 2016; 36(1):24-0.

18. Carvalho EV, Costa VVL, Araújo MS, Martens IBG, Sá NNB, Silva RA. Correlação entre o estado nutricional e o risco coronariano de adultos atendidos em uma ação educativa em Belém Pará. Revista Brasileira de Obesidade, Nutrição e Emagrecimento. 2016; 10(55):40-9.

19. Esperandio EM, Espinosa MM, Martins MSA, Guimarães LV, Lopes MAL, Scala LCN. Prevalência e fatores associados à hipertensão arterial em idosos de municípios da Amazônia Legal, MT. Rev. Bras. Geriatr. e Gerontol. 2013; 16(3):481-93.

20. Segundo VHO, Azevedo KPM, Rebouças GM, Felipe TR, Filho NJBA, Medeiros HJ, et al. Perfil nutricional e prevalência de hipertensão em idosos participantes de um programa de exercício físico. Rev Pesq Saúde. 2015; 16(1):7-0.

21. Stopa SR, César CLG, Segri NJ, Goldbaum M, Guimarães VMV, Alves MCGP, et al. Diabetes autorreferido em idosos: comparação das prevalências e medidas de controle. Rev Saúde Pública. 2014; 48(4):554-62. 
22. Neumann B, Conde SR, Lemos JRN, Moreira TR. Associação entre o estado nutricional e a prevalência de doenças crônicas não transmissíveis em idosos residentes no município de Roca Sales - RS. RBCEH. 2014; 11(2):16677.

23. Tavares DMS, Heitor SFD, Dias FA, Gomes NC, Rodrigues LR. Preditores de osteoporose entre idosos da área rural. REAS [Internet]. 2014; 3 (1): 14-5.

24. Sardinha AHL, Silva CG, Sena LB, Mesquita LLS, Rodrigues JB, Silva KNR. Adesão dos idosos com doenças crônicas ao tratamento medicamentoso. Rev Pesq Saúde. 2015; 16(30):154-8.

25. Brito MCC, Freitas CASL, Vasconcelos MIO, Dias MSA, Santiago LMM, Gomes DF. Atenção à saúde do idoso e família: evidências da produção científica. Revista Kairós Gerontologia. 2014;17(1):87-01. 